\title{
Simplification of Cylindrical Algebraic Formulas
}

\author{
Changbo Chen $^{\dagger}$, Marc Moreno Maza ${ }^{\dagger, \dagger}$ \\ $\dagger$ Chongqing Key Laboratory of Automated Reasoning and Cognition, Chongqing \\ Institute of Green and Intelligent Technology, Chinese Academy of Sciences \\ $\ddagger$ ORCCA, University of Western Ontario \\ changbo.chen@hotmail.com, moreno@csd.uwo.ca
}

\begin{abstract}
For a set $S$ of cells in a cylindrical algebraic decomposition of $\mathbb{R}^{n}$, we introduce the notion of generalized cylindrical algebraic formula (GCAF) associated with $S$. We propose a multi-level heuristic algorithm for simplifying the cylindrical algebraic formula associated with $S$ into a GCAF. The heuristic strategies are motivated by solving examples coming from the application of automatic loop transformation. While the algorithm works well on these examples, its effectiveness is also illustrated by examples from other application domains.
\end{abstract}

\section{Introduction}

Cylindrical algebraic decomposition (CAD), introduced by G. E. Collins [8], is a fundamental tool in real algebraic geometry. One of its main applications, also the initial motivation, is to solve quantifier elimination problems in the first order theory of real closed fields. Since its introduction, CAD has been improved by many authors and applied to numerous applications. The implementation of CAD is now available in different software, such as QEPCAD, Mathematica, Redlog, SyNRAC, RegularChains, and many others.

A CAD of $\mathbb{R}^{n}$ decomposes $\mathbb{R}^{n}$ into disjoint connected semi-algebraic sets, called cells, such that any two cells are cylindrically arranged, which implies that the projections of any two cells onto any $\mathbb{R}^{k}, 1 \leq k<n$, are either identical or disjoint. For a given semi-algebraic set $S$, one can compute a CAD $\mathcal{C}$ such that $S$ can be written as a union of cells in $\mathcal{C}$. Each cell is represented by a cylindrical algebraic formula (CAF) [14], whose zero set is the cell.

The CAF $\phi_{c}\left(x_{1}, \ldots, x_{n}\right)$ representing a CAD cell $c$ of $\mathbb{R}^{n}$ is a conjunction of finitely many atomic formulas of the form $x_{i} \sigma \operatorname{Root}_{x_{i}, k}(p)$, where $p \in$ $\mathbb{R}\left[x_{1}, \ldots, x_{i}\right]$ and $\operatorname{Root}_{x_{i}, k}(p)$ denotes the $k$-th real root (counting multiplicities) of $p$ treated as a univariate polynomial in $x_{i}$. The precise definition of CAF is given in Section 2 . The $\mathrm{CAF} \phi_{c}\left(x_{1}, \ldots, x_{n}\right)$ has a very nice property, namely the projection of $c$ onto $\mathbb{R}^{j}, 1 \leq j<n$, is exactly the zero set of the sub-formula of $\phi_{c}$, which is obtained by taking the conjunction of all atomic formulas in $\phi_{c}$ involving only the variables $x_{1}, \ldots, x_{j}$. Let $S$ be a set of cells $c_{1}, \ldots, c_{t}$ from a CAD $\mathcal{C}$. Denote by $\phi_{S}$ the zero set of $S$, thus we have $\phi_{S}:=\vee_{i=1}^{t} \phi_{c_{i}}$. The formula $\phi_{S}$ is also called a cylindrical algebraic formula. 
A CAF is a special extended Tarski formula, see [2]. While a Tarski formula is often the default output of quantifier elimination procedures, a CAF is also important for several reasons. Firstly, computing CAFs can be done by means of a CAD procedure without introducing additional augmented projection factors, which can bring substantial savings in terms of computation resources. Secondly, CAFs have a nice structure: the projection of a CAF onto any lower-dimensional space can be easily read off from the CAF itself, as mentioned before. Moreover, since a CAF is used to describe CAD cells, it naturally exhibits a polychotomous structure. This property is usually not true for Tarski formula output. Thirdly, each atomic formula of a CAF has the convenient format $x \sigma E$, where $E$ is an indexed root expression. This explicit expression is particularly useful in applications which care about the specific value of each coordinate, like in loop transformations of computer program $[12,11]$. Last but not least, performing set-theoretical operations on CAFs can be done efficiently, without explicit conversion to Tarski formulas [14]. This latter property supports an incremental algorithm for computing CADs [15].

While CAFs have many advantages, they have also their own drawbacks. Firstly, indexed root expressions are usually less handy to manipulate than polynomial expressions. This is because a polynomial function is defined everywhere while an indexed root expression is usually defined on a particular set. Secondly, due to numerous CAD cells being generated, a CAD-based QE solver usually outputs very lengthy CAFs, which could make the output formula not easy to use. For the particular application of loop transformation of computer programs, too many case distinctions might substantially increase the arithmetic cost of evaluating the transformed program as well as the number of misses in accessing cache memories. Therefore, simplification of CAFs is clearly needed. However, we have not seen much literature devoted to this topic except Chapter 8 of Brown's $\mathrm{PhD}$ thesis [2], The differences between Brown's approach and the one proposed is the present paper are discussed in Section 6. We remark that the Reduce command of Mathematica does perform some simplification before outputting CAFs. See Section 5 for an experimental comparison with Mathematica.

Since CAFs are generated from CAD cells, it is a natural idea to make use of the CAD data structure to simplify CAFs. In this paper, we produce a multi-level merging procedure for simplifying CAFs by exploiting structural properties of the $\mathrm{CAD}$ from which those CAFs are being generated. Although this procedure aims at improving the output of CAD solvers based on regular chains, it is also applicable to other CAD solvers. The merging procedure, presented formally in Section 4, consists of several reasonable and workable heuristics, most of which are motivated by solving examples taken from [12,7]. See Section 3 for details. The simplification procedure has four levels, where an upper level never produces more conjunctive clauses than the lower levels. The first two levels merge adjacent $\mathrm{CAD}$ cells, whereas the last two levels attempt to simplify a CAF into a single conjunctive clause, which is usually expected in the application of loop transformation. Thus the first two levels are expected to be effective for general $\mathrm{QE}$ problems whereas the last two are expected to be effective for $\mathrm{QE}$ 
problems arising from loop transformation. This expectation is justified also by the experimentation in Section 5.

The method has been implemented and new options are added to both the CylindricalAlgebraicDecompose and QuantifierElimination commands of the RegularChains library. The effectiveness of this algorithm is illustrated by examples in Sections 3 and 5. The experimentation shows that our heuristics work well. The running time overhead of simplification compared to the running time of the quantifier elimination procedure itself is negligible in the first two levels and acceptable in the advanced levels of the proposed heuristics.

There have already been a few works on the simplification of Tarski formulas, see for example $[10,4,3,13]$. Our work is concerned with the simplification of extended Tarski formulas, which allow indexed root expressions besides polynomial constraints. Moreover, the simplification goal here is to reduce as much as possible the number of conjunctive $\mathrm{CAF}$ clauses while still maintaining the feature of case distinctions. We emphasize that the motivation and the main targeting application of this work is to unify the CAFs generated in the application of loop transformation. In such applications, explicit bounds of loop indices are needed and the number of case distinctions is expected to be as small as possible in order to reduce the code size.

\section{Preliminary}

In this section, we first review the notion of cylindrical algebraic decomposition and cylindrical algebraic formula $(\mathrm{CAF})$. Then we define the notion of generalized CAF in order to represent the combination of CAFs.

Real algebraic function. Let $S \subset \mathbb{R}^{n}$. Let $f\left(x_{1}, \ldots, x_{n}, y\right) \in \mathbb{R}\left[x_{1}, \ldots, x_{n}, y\right]$. Let $k$ be a positive integer. Assume that for every point $\alpha$ of $S$, the univariate polynomial $f(\alpha, y)$ has at least $k$ real roots ordered by increasing value, counting multiplicities. Let $\operatorname{Root}_{y, k}(f)$ be a function which maps every point $\alpha$ of $S$ to the $k$-th real root of $f(\alpha, y)$. The function $\operatorname{Root}_{y, k}(f)$ is called a real algebraic function defined on $S$.

Stack over a semia-algebraic set. Let $S$ be a connected semi-algebraic subset of $\mathbb{R}^{n-1}$. The cylinder over $S$ in $\mathbb{R}^{n}$ is defined as $Z_{\mathbb{R}}(S):=S \times \mathbb{R}$. Let $\theta_{1}<\cdots<$ $\theta_{s}$ be continuous real algebraic functions defined on $S$. Denote $\theta_{0}=-\infty$ and $\theta_{s+1}:=\infty$. The intersection of the graph of $\theta_{i}$ with $Z_{\mathbb{R}}(S)$ is called the $\theta_{i}$-section of $Z_{\mathbb{R}}(S)$. The set of points between $\theta_{i}$-section and $\theta_{i+1}$-section, $0 \leq i \leq s$, of $Z_{\mathbb{R}}(S)$ is a connected semi-algebraic subset of $\mathbb{R}^{n}$, called a $\left(\theta_{i}, \theta_{i+1}\right)$-sector of $Z_{\mathbb{R}}(S)$. The sequence $\left(\theta_{0}, \theta_{1}\right)$-sector, $\theta_{1}$-section, $\left(\theta_{1}, \theta_{2}\right)$-sector, $\ldots, \theta_{s}$-section, $\left(\theta_{s}, \theta_{s+1}\right)$-sector form a disjoint decomposition of $Z_{\mathbb{R}}(S)$, called a stack over $S$, which is uniquely defined for given functions $\theta_{1}<\cdots<\theta_{s}$.

Cylindrical algebraic decomposition. Let $\pi_{n-1}$ be the standard projection from $\mathbb{R}^{n}$ to $\mathbb{R}^{n-1}$ mapping $\left(x_{1}, \ldots, x_{n-1}, x_{n}\right)$ onto $\left(x_{1}, \ldots, x_{n-1}\right)$. A finite partition $\mathcal{D}$ of $\mathbb{R}^{n}$ is called a cylindrical algebraic decomposition (CAD) of $\mathbb{R}^{n}$ if one of the following properties holds. 
- either $n=1$ and $\mathcal{D}$ is a stack over $\mathbb{R}^{0}$,

- or the set of $\left\{\pi_{n-1}(D) \mid D \in \mathcal{D}\right\}$ is a CAD of $\mathbb{R}^{n-1}$ and each $D \in \mathcal{D}$ is a section or sector of the stack over $\pi_{n-1}(D)$.

When this holds, the elements of $\mathcal{D}$ are called cells. The set $\left\{\pi_{n-1}(D) \mid D \in \mathcal{D}\right\}$ is called the induced CAD of $\mathcal{D}$. A CAD $\mathcal{D}$ of $\mathbb{R}^{n}$ can be encoded by a tree, called a CAD tree (denoted by $T$ ), as below. The root node, denoted by $r$, is $\mathbb{R}^{0}$. The children nodes of $r$ are exactly the elements of the stack over $\mathbb{R}^{0}$. Let $T_{n-1}$ be a CAD tree of the induced CAD of $\mathcal{D}$ in $\mathbb{R}^{n-1}$. For any leaf node $C$ of $T_{n-1}$, its children nodes are exactly the elements of the stack over $C$.

Cylindrical algebraic formula. Let $c$ be a cell in a CAD of $\mathbb{R}^{n}$. A cylindrical algebraic formula associated with $c$, denoted by $\phi_{c}$, is defined recursively.

(i) The case for $n=1$. If $c=\mathbb{R}$, then $\phi_{c}:=$ true. If $c$ is a point $\alpha$, then define $\phi_{c}:=x_{1}=\alpha$. If $c$ is an open interval $(\alpha, \beta) \neq \mathbb{R}$, then $\phi_{c}:=c>\alpha \wedge c<\beta$. For the special case that $\alpha=-\infty$, then $\phi_{c}$ is simply written as $c<\beta$. Similarly if $\beta=+\infty, \phi_{c}$ is simply written as $c>\alpha$.

(ii) The case for $n>1$. Let $c_{n-1}$ be the projection of $c$ onto $\mathbb{R}^{n-1}$. If $c=c_{n-1} \times \mathbb{R}$, then define $\phi_{c}:=\phi_{c_{n-1}}$. If $c$ is an $\theta_{i}$-section, then $\phi_{c}:=\phi_{c_{n-1}} \wedge x_{n}=\theta_{i}$. If $c$ is an $\left(\theta_{i}, \theta_{i+1}\right)$-sector, then $\phi_{c}:=\phi_{c_{n-1}} \wedge x_{n}>\theta_{i} \wedge x_{n}<\theta_{i+1}$. If $\theta_{i}=-\infty$, then $\phi_{c}$ is simply written as $\phi_{c_{n-1}} \wedge x_{n}<\theta_{i+1}$. If $\theta_{i+1}=+\infty$, then $\phi_{c}$ is simply written as $\phi_{c_{n-1}} \wedge x_{n}>\theta_{i}$.

If $\phi_{c}$ is the CAF associated with $c$, its zero set is defined as $Z_{\mathbb{R}}\left(\phi_{c}\right):=c$. Let $S$ be a set of disjoint cells in a CAD. If $S=\emptyset, \phi_{S}:=$ false. Otherwise, a CAF associated with $S$ is defined as $\phi_{S}:=\vee_{c \in S} \phi_{c}$. Its zero set is $Z_{\mathbb{R}}\left(\phi_{S}\right):=\cup_{c \in S} c$.

Example 1 Consider the closed unit disk $S$ defined by $x^{2}+y^{2} \leq 1$. Then a CAF associated with $S$ is as below.

$$
\begin{aligned}
(x=-1 \wedge y=0) & \vee\left(-1<x \wedge x<1 \wedge y=-\sqrt{1-x^{2}}\right) \\
& \vee\left(-1<x \wedge x<1 \wedge-\sqrt{1-x^{2}}<y \wedge y<\sqrt{1-x^{2}}\right) \\
& \vee\left(-1<x \wedge x<1 \wedge y=\sqrt{1-x^{2}}\right) \\
& \vee(x=1 \wedge y=0)
\end{aligned}
$$

Extended Tarski formula [2]. A (restricted) extended Tarski formula (ETF) is a Tarski formula, with possibly the addition of atomic formulas of the form $x_{i} \sigma \operatorname{Root}_{x_{i}, k}(f)$, where $\operatorname{Root}_{x_{i}, k}(f), 1 \leq i \leq n$, is a real algebraic function (defined on some set), and $\sigma \in\{=, \neq,>,<, \geq, \leq\}$. Given an ETF $\Phi$, we can always write it in a disjunctive normal form $\Phi=\vee_{i=1}^{s} \wedge_{j=1}^{s_{i}} \phi_{i, j}$. Let $\Phi_{i}:=$ $\wedge_{j=1}^{s_{i}} \phi_{i, j}$. Assume that the variables $x_{1}, \ldots, x_{n}$ are ordered as $x_{1}<\cdots<x_{n}$. Let $v\left(\phi_{i, j}\right)$ be the biggest variable appearing in $\phi_{i, j}$. Then we can always arrange the order of atomic formulas appearing in each $\Phi_{i}$ such that for any $\phi_{i, j_{1}}$ and $\phi_{i, j_{2}}$, where $j_{1}<j_{2}$, we have $v\left(\phi_{i, j_{1}}\right) \leq v\left(\phi_{i, j_{2}}\right)$. Let $w \in\left\{x_{1}, \ldots, x_{n}\right\}$. Denote by $\Phi_{i}^{<w}:=\wedge_{v\left(\phi_{i, j}\right)<w} \phi_{i, j}$. We say $\Phi_{i}$ is proper if for any $j=1, \ldots, s_{i}$, if $\phi_{i, j}=$ $v \sigma \operatorname{Root}_{v, k}(f)$, then $\operatorname{Root}_{v, k}(f(\alpha))$ is defined for all $\alpha$ satisfying $\Phi_{i}^{<v}$. We say $\Phi$ is proper if every $\Phi_{i}$ is proper. It is clear that a CAF is a proper restricted ETF.

Generalized Cylindrical Algebraic Formula (GCAF). Let $S$ be a set of disjoint cells in a CAD of $\mathbb{R}^{n}$. A GCAF associated with $S$, denoted by $\Phi$, is a proper restricted ETF $\Phi=\vee_{i=1}^{s} \wedge_{j=1}^{s_{i}} \phi_{i, j}$ such that 
- the zero set of $\Phi$ is exactly $\cup_{c \in S} c$,

- the zero set of $\Phi_{i}:=\wedge_{j=1}^{s_{i}} \phi_{i, j}$ is a union of some cells in $S$,

- the zero sets of $\Phi_{i}$ and $\Phi_{j}$ are disjoint for $1 \leq i<j \leq s$,

- each $\phi_{i, j}$ is of the form $v=\operatorname{Root}_{v, k}(f)$, where $v \in\left\{x_{1}, \ldots, x_{n}\right\}$,

- for every $w \in\left\{x_{1}, \ldots, x_{n}\right\}$, we have $\pi_{<w}\left(\Phi_{i}^{\leq w}\right)=\left(\Phi_{i}^{<w}\right)$, where $\Phi_{i}^{\leq w}:=$ $\wedge_{v\left(\phi_{i, j}\right) \leq w} \phi_{i, j}$ and $\Phi_{i}^{<w}:=\wedge_{v\left(\phi_{i, j}\right)<w} \phi_{i, j}$.

A GCAF clearly has a cylindrical structure justifying its name. Note that both a CAF and a GCAF can be naturally encoded in a tree data structure, with each node representing an atomic formula $\phi_{i, j}$. This tree together with the CAD cells information is used in algorithms for simplifying CAFs in Section 4.

Example 2 Both $\left(-1 \leq x \wedge x \leq 1 \wedge-\sqrt{1-x^{2}} \leq y \wedge y \leq \sqrt{1-x^{2}}\right)$ and

$$
\begin{aligned}
(x=-1 \wedge y=0) & \vee\left(-1<x \wedge x<1 \wedge-\sqrt{1-x^{2}} \leq y \wedge y \leq \sqrt{1-x^{2}}\right) \\
& \vee(x=1 \wedge y=0)
\end{aligned}
$$

are GCAFs equivalent to the CAF in Example 1.

The simplification procedure presented in this paper turns a CAF $\phi$ (in disjunctive normal form) into an equivalent GCAF $\Phi$ (in disjunctive normal form). We say $\Phi$ is simpler than $\phi$ if the number of conjunctive clauses in $\Phi$ is strictly less than that of $\phi$.

\section{Motivating examples}

In this section, we go through several examples, coming from the application of automatic loop transformation, so as to introduce the heuristic strategies for combing CAFs. Those strategies are formally presented in Section 4. We refer the reader to $[12,7]$ for the application background of these examples.

Example 3 We consider polynomial multiplication with synchronous scheduling. It is formulated as the following quantifier elimination problem. If no simplification is applied, the result consists of 10 conjunctive clauses, as shown below.

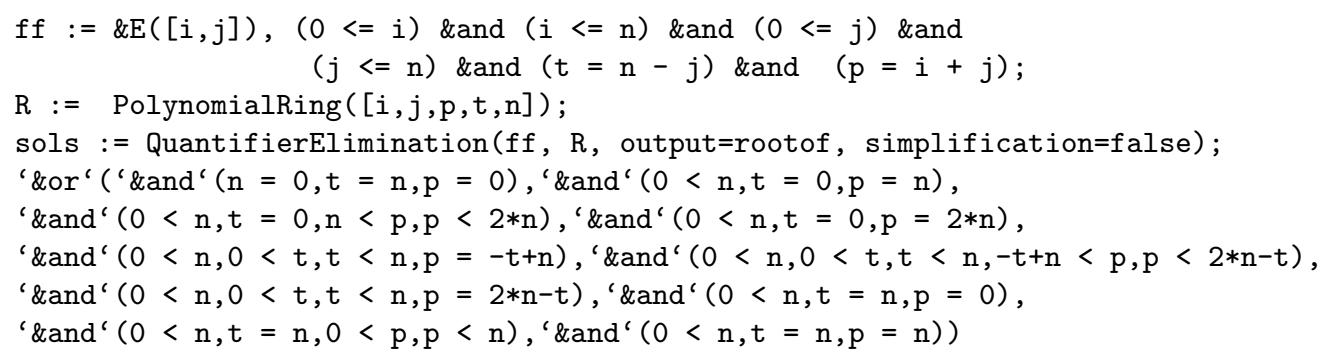

We observe that some conjunctive clauses can be merged into one. For instance, consider the subformula

$$
\begin{gathered}
(0<n \wedge t=0 \wedge p=n) \\
\vee(0<n \wedge t=0 \wedge n<p \wedge p<2 n) . \\
\vee(0<n \wedge t=0 \wedge p=2 n)
\end{gathered}
$$


Note that $(0<n \wedge t=0)$ is common to all three conjunctive clauses. Applying the distributivity law, the above formula is equivalent to

$$
(0<n \wedge t=0) \wedge((p=n) \vee(n<p \wedge p<2 n) \vee(p=2 n)) .
$$

Observe that $(p=n) \vee(n<p \wedge p<2 n) \vee(p=2 n)$ can be combined into one conjunctive clause, namely $n \leq p \wedge p \leq 2 n$. Thus, the above sub-formula of Equation (1) is equivalent to

$$
0<n \wedge t=0 \wedge n \leq p \wedge p \leq 2 n .
$$

The above transformation can be explained in the language of CAD. Here $(0<$ $n \wedge t=0$ ) represents a CAD cell of $\mathbb{R}^{2}$ while $p=n, n<p \wedge p<2 n$, and $p=2 n$ represent respectively three adjacent children of it, which makes the combination straightforward. This observation forms our first idea. Applying this strategy to the entire expression in Equation (1) yields the following simplified formula:

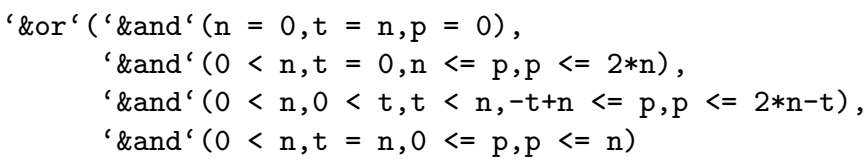

Let us look at the last three conjunctive clauses. At first glance, it seems that they cannot be combined into one. A key observation is that if we specialize $-t+n \leq p \wedge p \leq 2 n-t$ at $t=0$ and $t=n$, we obtain $n \leq p \wedge p \leq 2 n$ and $0 \leq p \wedge p \leq n$. Thus the last three conjunctive clauses can be combined into one:

'\&and' $(0<\mathrm{n}, 0<=\mathrm{t}, \mathrm{t}<=\mathrm{n},-\mathrm{t}+\mathrm{n}<=\mathrm{p}, \mathrm{p}<=2 * \mathrm{n}-\mathrm{t})$.

Applying this specialization technique again, we obtain the final output:

'\&and' $(0<=n, 0<=\mathrm{t}, \mathrm{t}<=\mathrm{n},-\mathrm{t}+\mathrm{n}<=\mathrm{p}, \mathrm{p}<=2 * \mathrm{n}-\mathrm{t})$.

Combining together the two simplification techniques introduced in Example 3 forms the basis for the first level of our simplification procedure. Here is the result produced by the QuantifierElimination command of the RegularChains library at level L1:

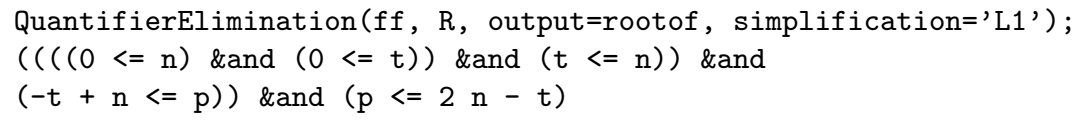

Example 4 In this example, we consider polynomial multiplication with asynchronous scheduling. The following shows the output without using simplification. The output has 12 conjunctive clauses.

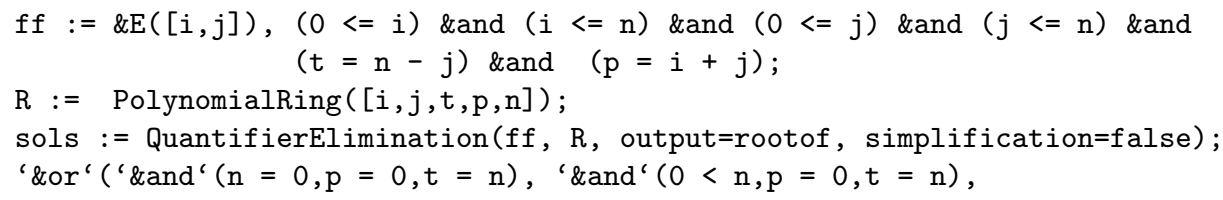




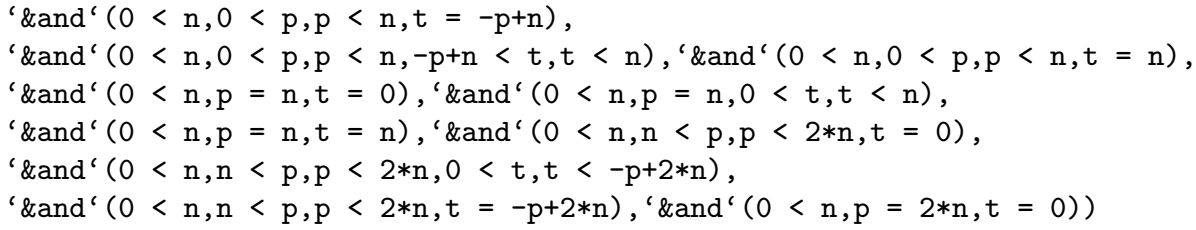

Here is the result produced by the QuantifierElimination command of the RegularChains library with the simplification level set to L1:

$(((n=0)$ \&and $(\mathrm{p}=0))$ \&and $(\mathrm{t}=0))$ \&or $(((((0<\mathrm{n})$ \&and $(0<=\mathrm{p}))$

\&and $(p<=n))$ \&and $(-p+n<=t))$ \&and $(t<=n)))$ \&or $(((((0<n)$

\&and $(n<p))$ \&and $(p<=2 n))$ \&and $(0<=t))$ \&and $(t<=-p+2 n))$

The situation here is a bit interesting. It seems that it is impossible to combine the three conjunctive clauses into one. This is because to make $-p+n \leq t \wedge t \leq$ $n \Longleftrightarrow 0 \leq t \wedge t \leq-p+2 n$ hold, we must have $-p+n=0$ and $-p+2 n=\bar{n}$, that is $n=p$ must hold. This obviously does not always hold under either the condition $0<n \wedge 0 \leq p \wedge p \leq n$ or the condition $0<n \wedge n<p \wedge p \leq 2 n$.

However, if we look more closely at the example, we find that the formulas

$\forall n, p, t \quad 0<n \wedge 0 \leq p \wedge p \leq n \wedge-p+n \leq t \wedge t \leq n \Longrightarrow 0 \leq t \wedge t \leq-p+2 n$.

and

$\forall n, p, t \quad 0<n \wedge n<p \wedge p \leq 2 n \wedge 0 \leq t \wedge t \leq-p+2 n \Longrightarrow-p+n \leq t \wedge t \leq n$.

are always true. Thus the last two can be combined into one

$$
0<n \wedge 0 \leq p \wedge p \leq 2 n \wedge-p+n \leq t \wedge t \leq n \wedge 0 \leq t \wedge t \leq-p+2 n
$$

This third simplification technique forms the foundation of option 3 of the algorithm presented in the next section. Now, the simplified output consists of the following single conjunction: The following is the simplified output.

$((((((0<=n)$ \&and $(0<=p))$ \&and $(p<=2 n))$ \&and $(n-p<=t))$ \&and $(t<=n))$ \&and $(0<=t))$ \&and $(t<=2 n-p)$

Example 5 Consider a more advanced example from [7]. Without simplification, the output cannot be displayed completely. Below, we only display the first 2 and the last 2 conjunctive clauses. There are 223 conjunctive clauses, in total.

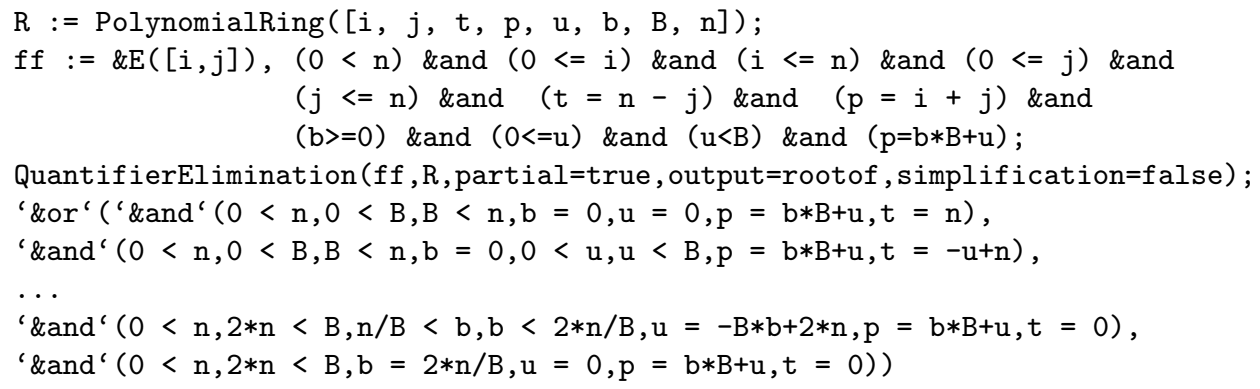


If the first two simplification techniques (options 1 or 2 in Section 4) are used, there are 29 conjunctive clauses. We only display the first 2 and the last 2 ones.

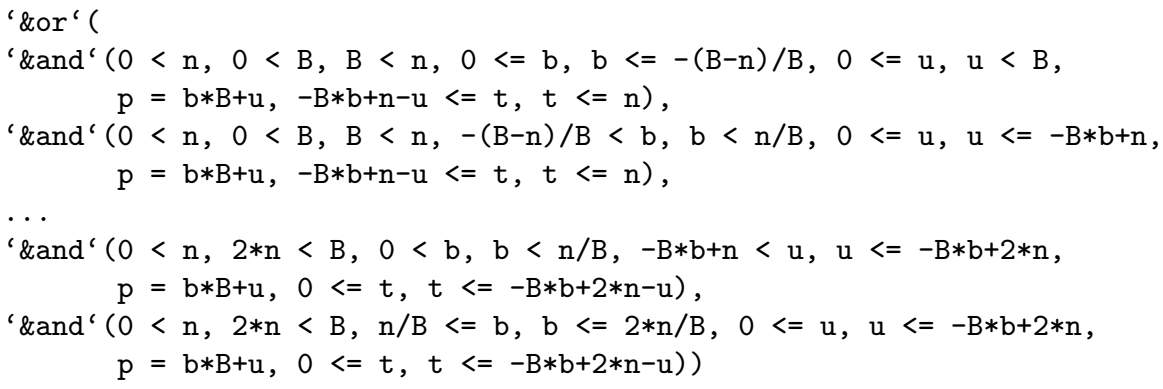

If we use Option 3, the output consist only 5 conjunctive clauses:

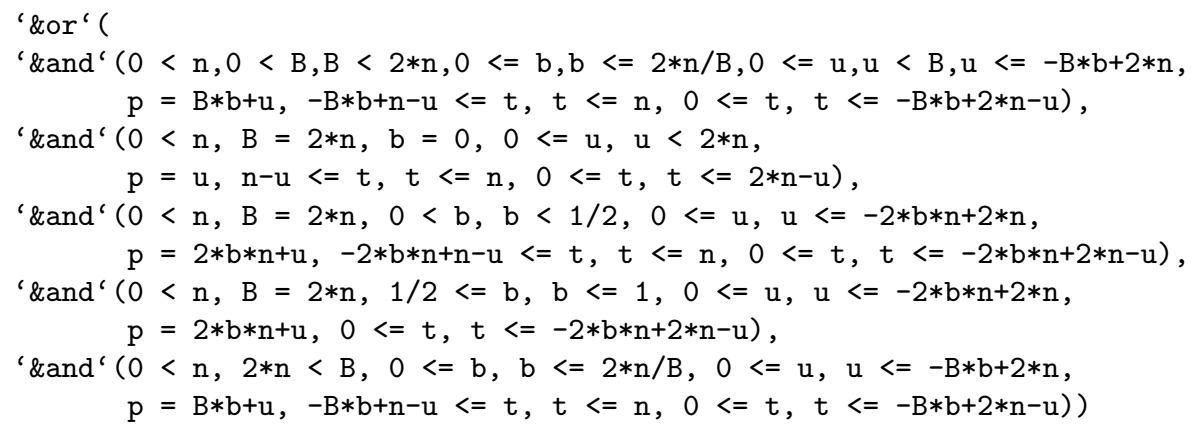

The situation here is more subtle. It can be shown that the third one and the fourth one can be combined together using the technique shown in option 3. But it can not be combined with the second one by specialization. This is because in the second one we have $0 \leq u \wedge u<2 n$ while in the third one we have $0 \leq u \wedge u \leq-2 b n+2 n$. When $\bar{b}=0$, the latter is equivalent to $0 \leq u \wedge u \leq 2 n$. So a more general "pivot formula" is needed, which can be found in the first and the fifth conjunctive clauses. The technical details are covered in Section 4. This idea form the basis of option 4 , which now brings a single conjunctive clause.

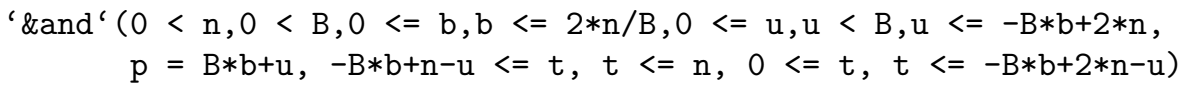

\section{Algorithm}

In this section, we present a heuristic algorithm Merge for combining cylindrical algebraic formulas, motivated by the examples shown in last section. In our algorithm, we have several levels of simplification. The most advanced level requires to decide the truth value of a quantifier free formula, which can be accomplished by a special QE procedure such as the one in [5] for computing triangular decomposition of semi-algebraic systems. In the following, we provide the pseudo 
code for the algorithm and explain the subroutines in detail. The explanation supplies a loose proof of the correctness of the algorithms.

The function Merge takes a CAF tree $T$ as input and returns an equivalent GCAF tree $T$. In below, we say a child node is even if it represents a section cell and odd if it represents a sector cell. Merge is called recursively to merge its odd children subtree first. The reason for doing this is that the representation of the odd nodes might be used as a reference to merge the even nodes. At last, it calls BasicMerge to merge the children of $r$, each of which is a rooted GCAF tree.

In BasicMerge, one type of subtree is treated in a special manner. It is a subtree rooted at a node $c$ in $T$ consisting of a single path. Let $\Gamma$ be such a subtree of height $h$. It can be represented by a list of nodes $\left\{c_{0}, c_{1}, \ldots, c_{h}\right\}$, where $c_{0}:=c$ and $c_{i}$ is the child of $c_{i-1}, i=1, \ldots, h$. Each $c_{i}$ is uniquely identified by an atomic formula $\psi\left(c_{i}\right)$ in a CAD tree data structure. In the following subroutines, we use c.nodeRep to denote $\psi\left(c_{0}\right)$, c.rep to denote $\wedge_{i=0}^{h} \psi\left(c_{i}\right)$, c.desRep to denote $\wedge_{i=1}^{h} \psi\left(c_{i}\right)$, and c.desDesRep to denote $\wedge_{i=2}^{h} \psi\left(c_{i}\right)$. Denote by c.ancRep the conjunction of $c$ 's ascendants' representing atomic formulas.

In the context of the subroutines, these objects are all well defined. The top level function Merge always compresses such a tree $\Gamma$ into a node. The procedure starts by dividing children of $r$ into blocks s.t. children in different blocks are not adjacent. If opt is 1, the child node which has children is not processed. If opt is 3 or 4 , the procedure tries to combine all the children nodes in a block into one. If it fails, opt 2 is adapted to combine as much adjacent children nodes in a block as possible. Note that we can also merge the nodes of the type $x_{i}<\operatorname{Root}_{x_{i}, k} f$ and $x_{i}>\operatorname{Root}_{x_{i}, k} f$ into one $x_{i} \neq \operatorname{Root}_{x_{i}, k} f$ even the two nodes are not adjacent. This justifies the call of IneqMerge.

The procedure BlockMergable is used to test if the children of $r$ in a block can be merged into one. Note that this function only handle the case that none of the children nodes in the block have children. To merge the children nodes which themselves have children, extra cost might be paid, since each subtree rooted at a child node is for sure not a tree consisting of a single path. The conjunction of the odd children is selected first as a pivot. If it fails and opt is 4 , the conjunction of odd siblings of $r$ is selected as a pivot.

When the procedure BlockMergable fails, the function NextMergable takes over to merge adjacent nodes in a block incrementally, which calls SameDesRep to test if the representation of the descendants of two adjacent nodes are equivalent. The equivalence is conducted by some simple test. In the case that the adjacent nodes have children, one checks if the subtrees rooted at them have physically the same representation by calling ExactSameDesRep. Otherwise, some simple specialization is conducted to check the equivalence.

\section{Experimentation}

In this section, we apply the algorithms of Section 4 to more examples. A comparison with the Reduce command of Mathematica is also provided. 


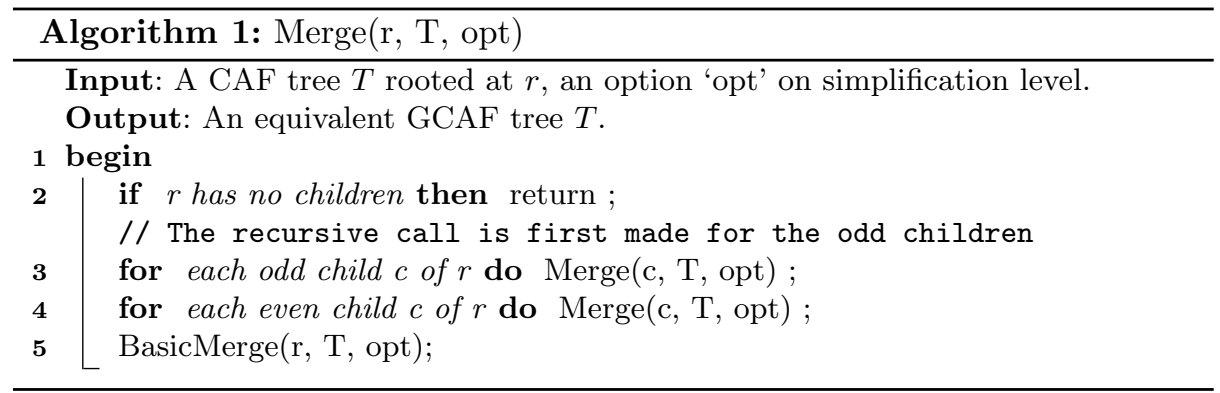

Example 6 [11] This example is about scanning index sets generated by applying a non-linear schedule. Without simplification, the output has 58 clauses. With option 1, the output has 10 conjunctive clauses. With option 3 , the output has exactly one conjunctive clause, although it takes about 200 more seconds.

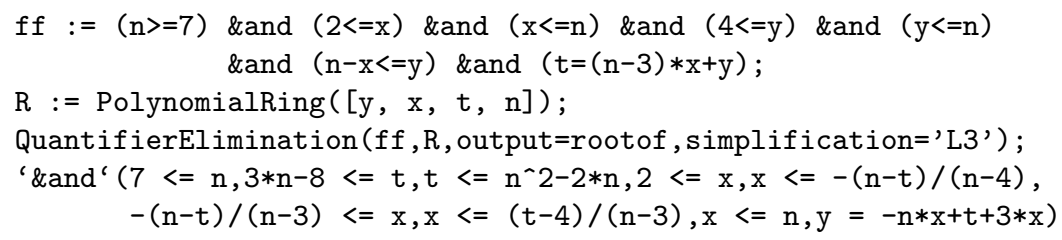

Example 7 [11] This example is about scanning index sets generated by normalizing loop strides. Without simplification, the output has 8 conjunctive clauses. The option 1 is sufficient to simplify it into one piece.

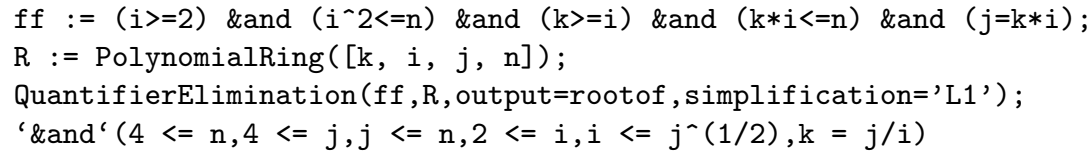

Example 8 [2] An example illustrating simple CAD. Without simplification, there are 51 clauses. After simplification with option 1, we have

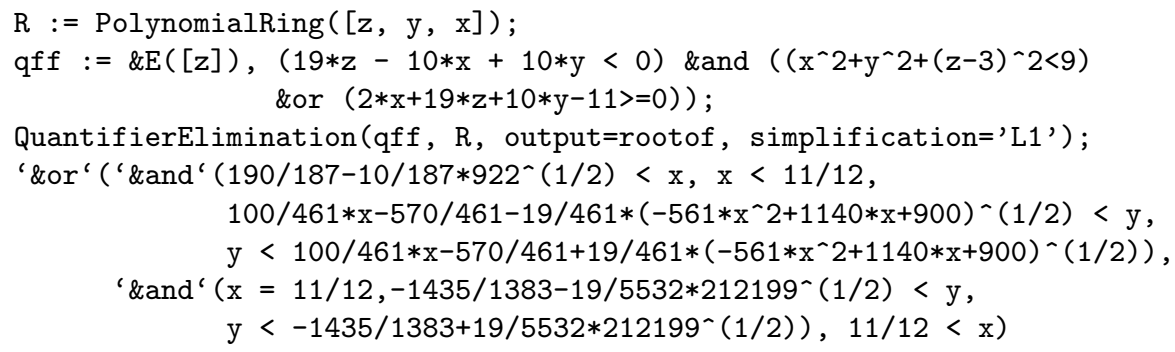

Example 9 An example from program termination analysis. Without simplification, the output has 246 conjunctive clauses. After simplification with option 2 , the output has 14 conjunctive clauses. 


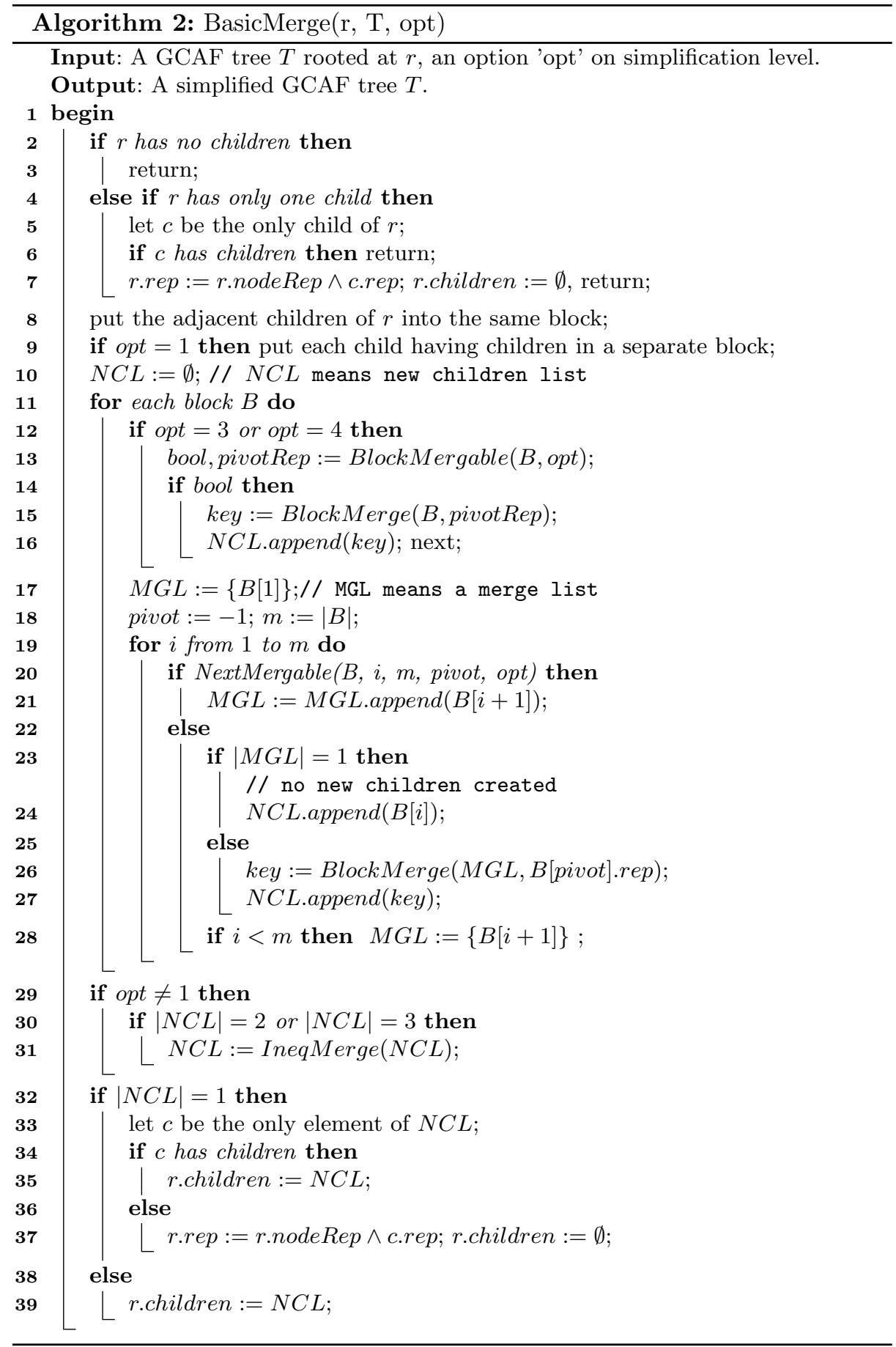



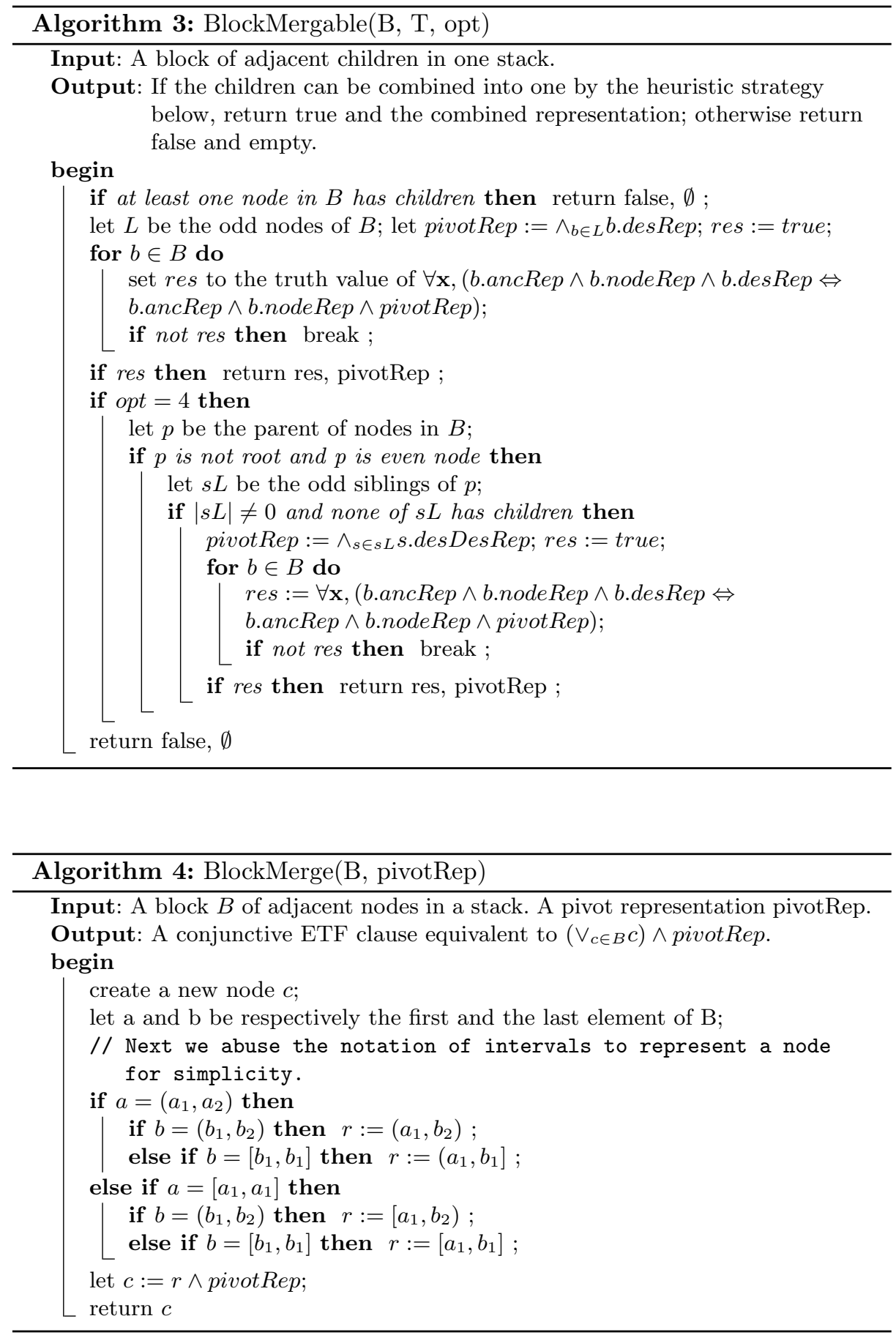

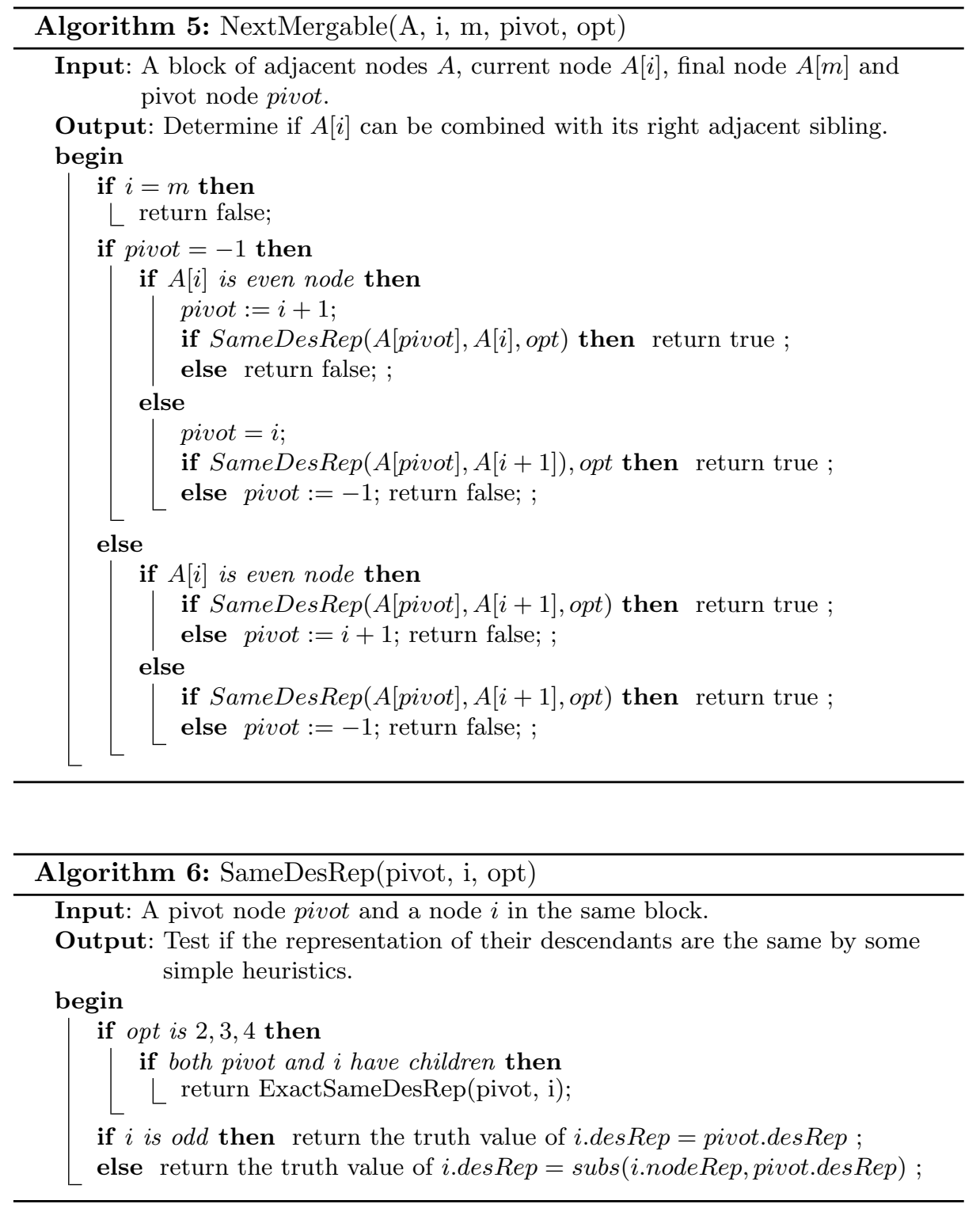

$\mathrm{R}:=$ PolynomialRing ([v1, v2, v3, labda, a11, a21, a22, a33, b12, b22, b23]);

$\mathrm{f}:=\& \mathrm{E}([\mathrm{v} 1, \mathrm{v} 2, \mathrm{v} 3, \mathrm{labda}]),(\mathrm{labda}>0)$ \&and $(\mathrm{a} 11 * \mathrm{v} 1=\mathrm{labda} * \mathrm{v} 1)$ \&and

$(\mathrm{a} 21 * \mathrm{v} 1+\mathrm{a} 22 * \mathrm{v} 2=\mathrm{labda} * \mathrm{v} 2)$ \&and $(\mathrm{a} 33 * \mathrm{v} 3=1 \mathrm{abda} * \mathrm{v} 3)$ \&and

(b12*v2>0) \&and (b22*v2+b23*v3>0);

QuantifierElimination ( $f, R$, output=rootof, partial=true, simplification='L2') ;

'\&or' ('\&and' (b23 <> 0, b22 < 0, b12 < 0, a22 <= 0, a21 $<0,0<$ a11),

'\&and' (b23 $<>0$, b22 $<0$, b12 $<0,0<a 22)$,

'\&and' (b23 <> 0, b22 < 0, $0<$ b12, $0<a 33, a 22<>a 33, a 21<>0, a 11=a 33$ ), 
tion of loop transformation, the levels L3 and L4 are effective. But for examples from other application domains, the level L2 is the best to choose considering the computation overhead, which is the default option of our QE procedure. W.r.t. the Reduce command, our QE procedure can generate less number of conjunctive clauses for 16 out of the 25 examples. For the three examples for which our QE procedure generates more conjunctive clauses, we have checked that the reason is that our routine ExactSameDesRep only checks whether two subtrees are physically the same while the Reduce command is more aggressive. Finding cost-effective heuristic strategies to handle this problem is left for future work.

\section{Conclusions}

We have presented a multi-level heuristic algorithm for simplifying cylindrical algebraic formulas, motivated by applications like automatic loop transformation in computer programs. The experimentation shows that the method can reduce significantly the number of conjunctive clauses of a CAF. Nevertheless, more work is required to obtain more compact output in less computing time. In particular, the following related work needs to be investigated. In Chapter 8 of his thesis [2], Brown presented algorithms for constructing cylindrical formulas, which are proper restricted extended Tarski formulas having cylindrical properties, but not necessarily GCAFs. An essential idea in his approach is the concept of "polynomially compatible". But it is not always easy to determine if two sections in different stacks are polynomially compatible, especially for cylindrical algebraic decomposition computed by the partial CAD approach [9] or the regular chain approach [6]. Moreover, the simplification techniques of [2] cannot handle the case taken care of by options 3 and 4 of our method. Nevertheless, the ideas of "polynomially compatible" and "truth-boundary cells" deserve to be investigated further and related to our approach. The adjacency algorithm [1] might also help determining whether two intro-stack sections can be combined.

Acknowledgments. Supported by the NSFC (11301524,11471307,61202131).

\section{References}

1. D. S. Arnon, G. E. Collins, and S. McCallum. Cylindrical algebraic decomposition II: an adjacency algorithm for the plane. SIAM J. Comput., 13(4):878-889, 1984.

2. C. W. Brown. Solution Formula Construction for Truth Invariant CAD's. PhD thesis, University of Delaware, 1999.

3. C. W. Brown. Fast simplifications for tarski formulas based on monomial inequalities. Journal of Symbolic Computation, 47(7):859 - 882, 2012.

4. C. W. Brown and A. Strzeboński. Black-box/white-box simplification and applications to quantifier elimination. In Proc. of ISSAC '10, pages 69-76, 2010.

5. C. Chen, J. H. Davenport, J. May, M. Moreno Maza, B. Xia, and R. Xiao. Triangular decomposition of semi-algebraic systems. In S.M. Watt, editor, Proceedings ISSAC 2010, pages 187-194, 2010.

6. C. Chen and M. Moreno Maza. An incremental algorithm for computing cylindrical algebraic decompositions. Computer Mathematics: Proc. of ASCM '12, pages 199$222,2014$. 


\begin{tabular}{|c|c|c|c|c|c|c|c|c|c|c|c|c|}
\hline Example & \multicolumn{2}{|c|}{ L0 } & \multicolumn{2}{|l|}{ L1 } & \multicolumn{2}{|l|}{ L2 } & \multicolumn{2}{|l|}{ L3 } & \multicolumn{2}{|l|}{ L4 } & \multicolumn{2}{|c|}{ Reduce } \\
\hline ArcSin-B & 0.899 & 10 & 1.192 & 8 & 0.986 & 6 & 1.004 & 6 & 0.983 & 6 & 0.096 & 12 \\
\hline A-Real-Implicitization & 0.977 & 6 & 1.021 & 5 & 1.013 & 2 & 1.264 & 2 & 1.309 & 2 & 0.069 & 5 \\
\hline Collision-A-from-B-H & 2.340 & 13 & 2.344 & 1 & 2.273 & 1 & 2.328 & 1 & 2.374 & 1 & 0.180 & 1 \\
\hline Cyclic-4 & 0.851 & 2 & 0.868 & 2 & 0.880 & 1 & 0.874 & 1 & 0.861 & 1 & 0.055 & 2 \\
\hline Edges-Square-Product & 57.378 & 249 & 57.507 & 5 & 58.158 & 5 & 58.188 & 5 & 58.668 & 5 & 4.753 & 7 \\
\hline Ellipse-A-from-B-H & 2.305 & 12 & 2.352 & 2 & 2.345 & 2 & 2.439 & 2 & 2.376 & 2 & 0.400 & 2 \\
\hline Intersection-dagger- $\mathrm{B}$ & 1.797 & 113 & 1.837 & 84 & 1.810 & 78 & 2.160 & 78 & 2.145 & 78 & 0.289 & 104 \\
\hline Kahan-A & 1.034 & 17 & 1.414 & 10 & 1.107 & 10 & 1.129 & 10 & 1.113 & 10 & 0.116 & 10 \\
\hline Kahan-B & 1.056 & 13 & 1.135 & 9 & 1.152 & 9 & 1.133 & 9 & 1.136 & 9 & 0.145 & 9 \\
\hline Loop- & 1.418 & 8 & 1.399 & 1 & 1.414 & 1 & 1.402 & 1 & 1.441 & 1 & 0.059 & 2 \\
\hline Loop-Termination & 44.383 & 246 & 43.795 & 186 & 44.128 & 14 & 44.215 & 14 & 44.547 & 14 & 0.161 & 58 \\
\hline $\mathrm{NL}-\mathrm{Sc}$ & 2.915 & 58 & 3.039 & 10 & 3.018 & 10 & 222.654 & 1 & 236.145 & 1 & 0.073 & 12 \\
\hline Off-Cent & 1.847 & 4 & 1.917 & 2 & 1.893 & 2 & 1.923 & 2 & 1.914 & 2 & 0.129 & 2 \\
\hline Parametri & 0.839 & 7 & 0.871 & 5 & 0.874 & 4 & 1.057 & 4 & 1.110 & 4 & 0.075 & 5 \\
\hline Poly-Mu & 1.191 & 12 & 1.232 & 3 & 1.257 & 3 & 1.672 & 1 & 1.692 & 1 & 0.054 & 6 \\
\hline Poly-1 & 0.959 & 10 & 1.028 & 1 & 1.015 & 1 & 1.379 & 1 & 1.360 & 1 & 0.052 & 2 \\
\hline Poly-M & 12.001 & 223 & 12.522 & 29 & 12.333 & 29 & 152.901 & 5 & 207.635 & 1 & 0.123 & 50 \\
\hline Positivity-of-Quartic & 1.480 & 10 & 1.497 & 4 & 1.575 & 3 & 1.473 & 3 & 1.468 & 3 & 0.094 & 4 \\
\hline Putnum-Example & 2.872 & 76 & 2.897 & 8 & 3.016 & 8 & 2.934 & 8 & 2.941 & 8 & 0.430 & 6 \\
\hline Random-dagger-A & 2.106 & 214 & 2.150 & 160 & 2.162 & 148 & 2.209 & 148 & 2.146 & 148 & 0.155 & 189 \\
\hline Range-of-Lower-Bounds & 1.057 & 2 & 1.121 & 1 & 1.119 & 1 & 1.091 & 1 & 1.157 & 1 & 0.075 & 1 \\
\hline Sphere-Half-Space & 2.297 & 9 & 2.319 & 3 & 2.298 & 3 & 2.302 & 3 & 2.423 & 3 & 0.076 & 2 \\
\hline Whitney-Umbrella & 0.908 & 8 & 0.964 & 7 & 1.003 & 3 & 1.257 & 3 & 1.455 & 3 & 0.066 & 7 \\
\hline $\mathrm{X}$-axis-Ellipse-Problem & 12.099 & 104 & 12.322 & 24 & 13.070 & 24 & 12.552 & 24 & 12.398 & 24 & 0.466 & 16 \\
\hline YangXia & 2.225 & 8 & 2.245 & 2 & 2.257 & 2 & 2.897 & 2 & 2.850 & 2 & 0.101 & 2 \\
\hline
\end{tabular}

Table 1. Comparison between different simplification options and Reduce.

7. C. Chen and M. Moreno Maza. Quantifier elimination by cylindrical algebraic decomposition based on regular chains. In Proc. of ISSAC '14, pages 91-98, 2014.

8. G. E. Collins. Quantifier elimination for real closed fields by cylindrical algebraic decomposition. Springer Lecture Notes in Computer Science, 33:515-532, 1975.

9. G. E. Collins and H. Hong. Partial cylindrical algebraic decomposition. Journal of Symbolic Computation, 12(3):299-328, 1991.

10. A. Dolzmann and T. Sturm. Simplification of quantifier-free formulas over ordered fields. Journal of Symbolic Computation, 24:209-231, 1995.

11. A. Größlinger. Scanning index sets with polynomial bounds using cylindrical algebraic decomposition. Number MIP-0803. 2008.

12. A. Größlinger, M. Griebl, and C. Lengauer. Quantifier elimination in automatic loop parallelization. J. Symb. Comput., 41(11):1206-1221, 2006.

13. H. Iwane, H. Higuchi, and H. Anai. An effective implementation of a special quantifier elimination for a sign definite condition by logical formula simplification. In $C A S C$, volume 8136 of $L N C S$, pages 194-208. 2013.

14. A. Strzeboński. Computation with semialgebraic sets represented by cylindrical algebraic formulas. In Proc. of ISSAC '10, pages 61-68. ACM, 2010.

15. A. Strzeboński. Solving polynomial systems over semialgebraic sets represented by cylindrical algebraic formulas. In Proc. of ISSAC '12, pages 335-342. ACM, 2012.

16. D. J. Wilson. Real geometry and connectedness via triangular description: Cad example bank, 2013. 